\title{
Design of Antenna with Low Intensity RFID Wireless Network Signal
}

\author{
Zheng Juan ${ }^{1}$ and Gu Juanjuan ${ }^{1}$ \\ ${ }^{1}$ Department of Electronics \& Electrical Engineering, Hefei University, \\ Hefei,Anhui, 230601, China \\ E-mail:zhengj@126.com
}

\begin{abstract}
Testing direction and relative distance nearest to test position of environmental field intensity are analyzed using simulating calculation, taking three-dimensional cube good conductor as general test equipment, under vertical incidence of single plane wave, the effect of electrical size of the test equipment on test point selection is analyzed; incident angle of plane wave is transformed to calculate an relative error between selected position field intensity and environmental field intensity in oblique incidence condition so as to meet the demand of the experiment; and then promote it to reverberation chamber which is distributed as superposed field of multiple columns plane wave, make an analytical calculation on relative error, which can be controlled within $3 \%$, of selected test point to satisfy the experimental requirements, and it can be applied to practical test.
\end{abstract}

Keywords: Reverberation chamber; plane wave; environmental filed intensity; antenna design; RFID

\section{Introduction}

By far, the field where effect test of electromagnetic environment is to be conducted includes open area, anechoic chamber or semi-anechoic chamber, TEM chamber, GTEM chamber and reverberation chamber. Transfer efficiency between electromagnetic excitation energy of test facility and radiation intensity is very low in open area and anechoic chamber etc. An radiator is installed in shielding case of high quality factor (Q) as electromagnetic excitation source for reverberation chamber, with the rotation of one or multiple metal mechanical agitators to change boundary conditions of electromagnetic field and resonance condition of shielding cavity, which will make electromagnetic field operate as a multi-mode way to achieve an electromagnetic environment of spatial statistical uniform, isotropy and random polarization so as to improve its homogeneity of field[1-3]. The structure and working principle of reverberation chamber determine its significant advantage compared with traditional test field, with relatively small driving power may motivate electromagnetic environment of higher strength, the formed random polarized environment may achieve effect measurement of electromagnetic radiation without making the equipment overturn or changing the antenna; laid down cost is low and test time is short; and it may accomplish intensity field test of radiated interference of a larger system.

Scattering of loaded object to electromagnetic field inside of reverberation will alter randomicity and uniformity of communication in reverberation chamber, which makes an impact on measurement of test equipment suffering from environmental field. The effect of antenna etc loaded object on filed distribution of reverberation chamber is analyzed by Hill.D.A and Calberg.U measuring average scattering cross section obtained of diminished sphere of $\mathrm{Q}$ value derivation and cylinder device in all incident angles and polarization direction integral [4-5], however, it does not involve any influence of test equipment of multi-cube shape on filed distribution of reverberation chamber. This paper 
will be begun with plane wave, set three-dimensional cube good conductor as general test equipment to analyze the effect of it on uniform field, put forward selective principle of an optimal test point under a certain error when characterization test equipment is suffering from environmental electric field intensity, and verify it in reverberation room where exist superposed field of random plane wave.

\section{Computation of Electromagnetics}

To achieve communication of RFID and take into consideration conditions such as low-power dissipation, rapid arithmetic speed, strong expansion capability and abundant on-chip resources etc, wireless communication module with short distance and low-bitrate design of CC2530 radio frequency chip, which supports IEEE 802.15.4 and ZigBee2007 protocol, is selected from TI company, CC2530 is a kind of chip that integrates MCU and RF inside, 256KB Flash and 8KB SRAM, and it may achieve 6Lowpan and CoAP. At the same time, by inserting UDP/IPv6 protocol stack in the node, wireless sensor network of traditional RFID is achieved based on network switch of smart RFID node, which makes the application of each device, may operate with Web service mode. Lithium battery of $3.7 \mathrm{~V} / 5000 \mathrm{~mA}$ is selected for power module to provide power for other modules, MCP73831 chip is selected for charge and discharge management module to conduct charging and discharging management and protection for power module to ensure that power module may continuously provide power supply for all modules, thus safeguarding an enduring normal operation of whole device. Sensor module mainly is temperature module DS18B20, which has a real-time detection of environmental temperature, realtime remaining shelf life is calculated according to prediction algorithm of remaining shelf life, after processing by device, LCD in the module will show it to users in digital form. Memory module may save log info in the local when device is out of connection so that the data will be submitted to server as it's connected again.

Test equipment is placed at test filed as loaded object, electricity and charge distribution formed result from interaction between charge particle of test equipment and incident electromagnetic field under electromagnetic wave radiation, as "secondary irradiation" results from "new field source" is electromagnetic scattering, will make an impact on measurement of field distribution. For computation of electromagnetic scattering, there are mainly three methods below: 1) rigorous solution of electromagnetic scattering. Mainly use method of separation of variables to solve analytical solution of scatterer of special shape, incident field is divided into multi-series form under a certain specific coordinate system, assume that scattered field can be expanded as similar multiseries form under this coordinate system, then integrate strict boundary conditions and uniqueness theorem of electromagnetic field, the expansion coefficient of scattered field determined is correct and an unique solution, it's applied to solve scatterer of vertical plane parallel or superposition for surface and coordinate; 2) approximate solution of electromagnetic scattering. According to electric size and suitable frequency of scatterer, there are mainly high-frequency approximation and low-frequency approximation methods, of which high-frequency approximation is primary approximate calculation method, it starts from local quality of field, reflecting field and scattered field are determined by local property of surface of incident field and scatterer, attention is needed to be paid on local area of scattering point on scatterer to attain a goal of simplifying correlation integral equation of scattered field, it's applied to analyze scattering characteristics of electrically large scatterer; 3) numerical solution of electromagnetic scattering mainly includes method of moment (MOM), finite element method (FEM) and Finite Difference Time Domain (FDTD) etc[6].

MOM is based on time domain integral equations of Stratton-Chu integral in electromagnetic theory, with electric charge $\rho$, magnetic charge $\rho^{\prime}$, electric current $J$ and magnetic current $J^{\prime}$ to solve scattered filed [6], in this article, Feko software whose 
make MOM as core algorithm is applied and good conductor cube is used as research object, the size is set as $1 \mathrm{~m}^{*} 1 \mathrm{~m}^{*} 1 \mathrm{~m}$, applying amplitude is an incident electromagnetism with $1 \mathrm{v} / \mathrm{m}$, incident plane wave with single unit scattering along $\mathrm{x}$ direction is used for inspecting scattered filed distribution nearby, thus seeking out the test point nearest to environmental field intensity.

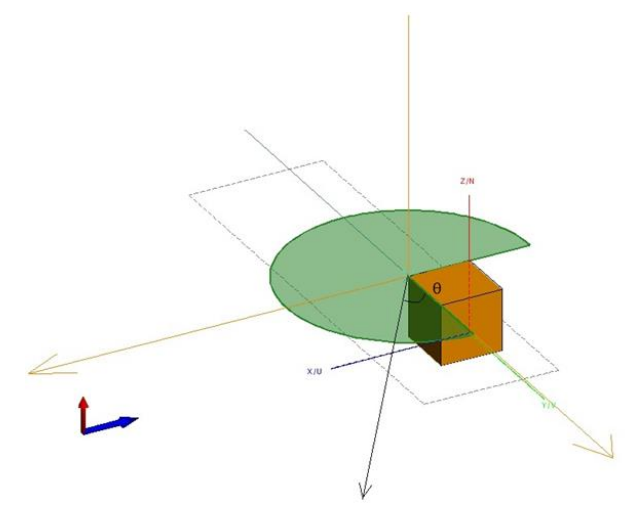

Figure 1. Test Angle $\theta$

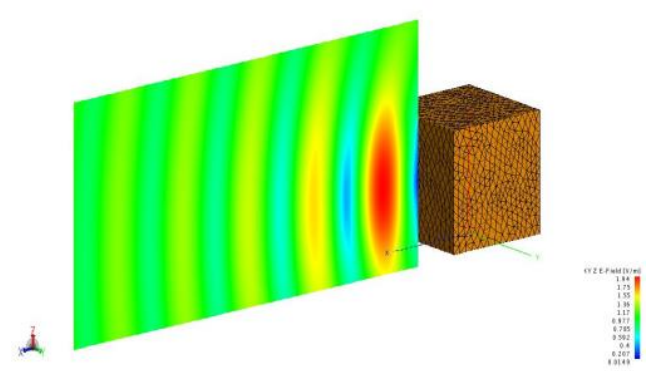

Figure 2. Adverse Scattered Field Distribution of Cube Scattering Direction and Irradiation Direction

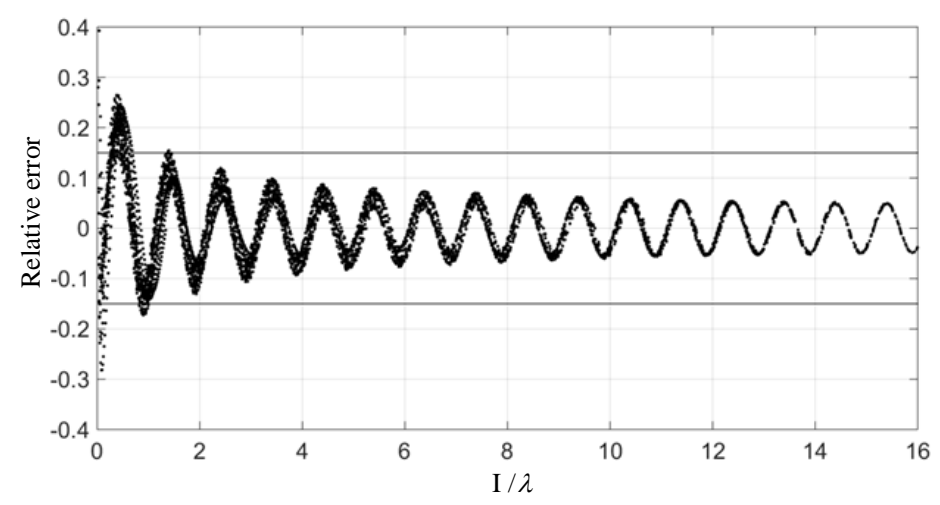

Figure 3. Variation of $\theta=180^{\circ}$ Relative Error along with Relative Distance

\section{Electromagnetic Field Space Distribution of Vertical Incidence of Single Plane Wave}

\subsection{Selection of Relative Distance for Test Point}

Assume that electromagnetic wave is an incidence along $\mathrm{x}$ direction, an included angle of measuring point and y-axis positive direction is $\theta$ (set clockwise as positive position 
as shown in figure 1), when $\theta \in\left(0^{\circ}, 90^{\circ}\right)$, scattered field direction of cube is alien to irradiation direction, and it generates standing wave distribution with incident field superposition of same frequency as shown in figure 2, it's not suitable for being the test point because of a greater test error. For experimental determination convenience, test method of $\theta=180^{\circ}$ is selected for inspecting relatively appropriate relative distance $l / \lambda$ of cube apex angle. Incident wave is also set as plane electromagnetic wave of continuously variable unit between $80 \mathrm{MHz}$ and $1 \mathrm{GHz}$, direction of polarization is along $\mathrm{Z}$-axis positive direction and incidence direction is along $\mathrm{X}$-axis, the incidence is from front end of vertical cube, compute electric field intensity of a distance between $0.05 \mathrm{~m}$ and $5 \mathrm{~m}$ and find the value of its module. 56 groups for frequency point are selected, 100 points for distance are selected as an interval of $0.05 \mathrm{~m}$, now $56^{*} 100$ group data are obtained, then process the data to get a distribution diagram in figure 3 , x-coordinate represents relative distance $l / \lambda, y$-coordinate represents corresponding relative measurement error, which is the difference value of filed intensity module value and incident field intensity and ratio of incident field intensity at test point. Figure 3 shows that a change rule of gradually damping vibration when relative error is enlarging along with relative distance, due to polarization current generated from when plane wave is irradiating the surface of good conductor, scattered field nearby the cube is radiation field of polarization current at this location, scattered radiation field is zero when the distance tends to infinite, then the field distribution at this location is electromagnetic field generated from plane wave and measuring error of field intensity module value tends to zero.

Figure 3 shows that when relative distance $l / \lambda \geq 2$, relative error both can be controlled within $\pm 15 \%$, however, for low-frequency working state, it's often hard to meet the demand of this kind of measuring distance. Figure 4.

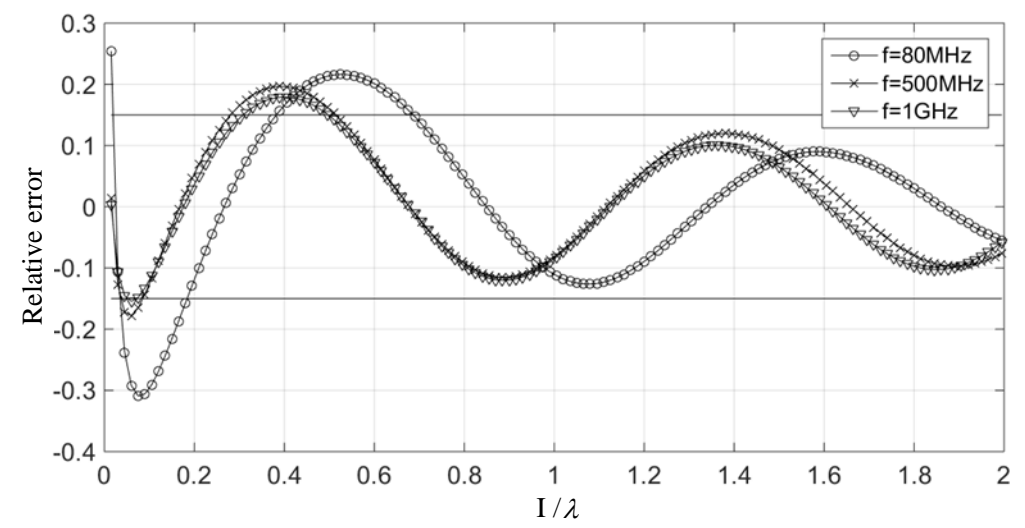

Figure 4. Variation Trend of Relative Error becomes Larger Along with Relative Distance when $f=80 \mathrm{MHz}, f=500 \mathrm{MHz}$ and $f=1 \mathrm{GHz}$ 


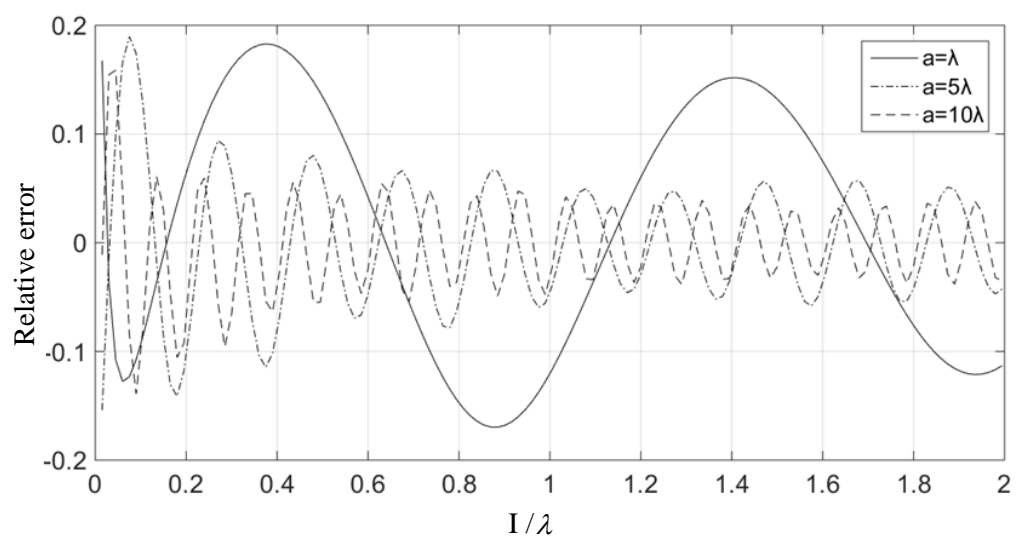

\section{Figure 5. Variation Tendency of Testing Relative Error Enlarges along with Relative Distance under Various Size when $f=300 \mathrm{MHz}$}

Relative distance $l / \lambda \in(0,2)$ is given, variation tendency of relative measurement error along with relative distance under working frequency of $f=80 \mathrm{MHz}, f=500 \mathrm{MHz}$ and $f=1 \mathrm{GHz}$, the higher working frequency, the lower relative error reduces, the figure shows that the relative distance $l / \lambda \in(0,0.1)$ when working frequency is in between of $80 \mathrm{MHz}$ and $1 \mathrm{GHz}$ and the first peak of relative error arises, relative error $l / \lambda \in(0.3,0.6)$ when the second peak arises, therefore, a maximum value, which is approximately $11 \%$, of relative error arises under $80 \mathrm{MHz}$ working frequency when relative distance $l / \lambda$ values 0.2 , when working frequency is in between of $80 \mathrm{MH}$ and $1 \mathrm{GHz}$, relative measurement error at this position is guaranteed within $\pm 15 \%$.

\subsection{Effect of Electrical Size of Test Equipment on Test Point Selection}

Change electrical size of the cube and set the side length of it as $\lambda 、 5 \lambda$ and $10 \lambda$, relative error of actual filed intensity module value and environmental field module value is measured as shown in figure 5, for different electrical size, $\theta=180^{\circ}$ test direction is selected under $300 \mathrm{MHz}$ working frequency, the variation tendency of relative distance enlarged along with relative distance is consistent, the field shows an damped oscillation variation, and relative error is within $\pm 15 \%$ when $l / \lambda=0.2$.

\section{Spatial Distribution of Incident Electromagnetic Field of Single Plane Wave}

Electromagnetic wave of vertical incidence and oblique incidence do exist when putting test equipment in reverberation chamber. Take conditions of non-normal incidence of incident plane wave into consideration, and assume that incident angle $\varphi$ of clockwise is positive direction, test direction selection is given in figure $6 \mathrm{a}, 6 \mathrm{~b}, 6 \mathrm{c}$ and $6 \mathrm{~d}$, when $\theta=180^{\circ}$ incident angle is $-30^{\circ},-60^{\circ}, 30^{\circ}$ and $60^{\circ}, 56$ frequency points between $80 \mathrm{MH}$ and $1 \mathrm{GHz}$ are selected to compute the variation of absolute value of measuring point relative error along with relative distance. Due to test direction selected is not necessarily the minimum direction of relative error when oblique incidence occurs, relative error and vertical incidence are rather bigger than the whole, however, data analysis in the figure shows that the relative error with selection of $\theta=180^{\circ}$ test direction and $l / \lambda=0.2$ may be guaranteed within $40 \%$, which meets the demand of experimental error. 


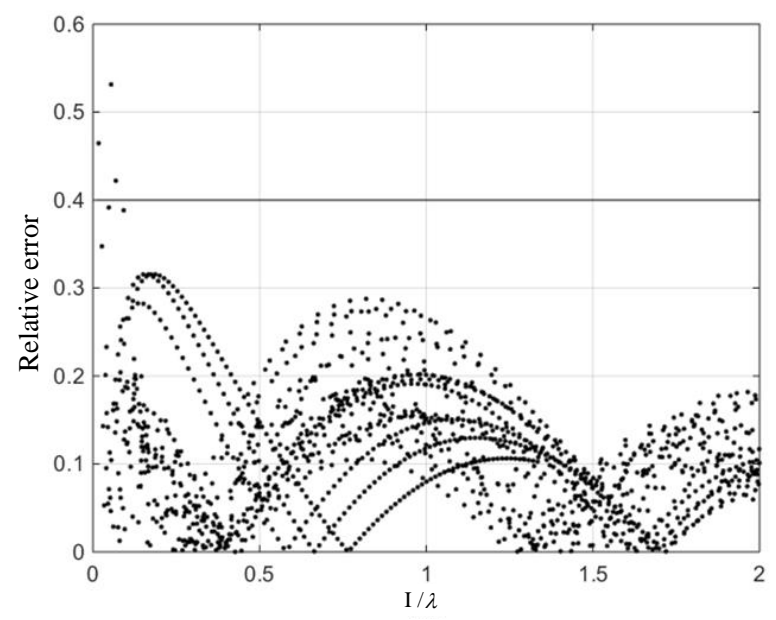

(a) $\varphi=-30^{\circ}$

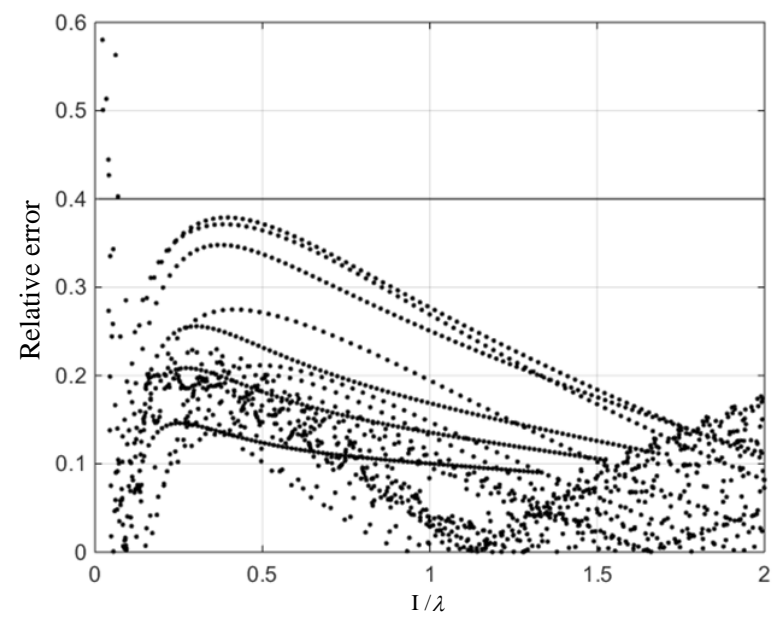

(b) $\varphi=-60^{\circ}$

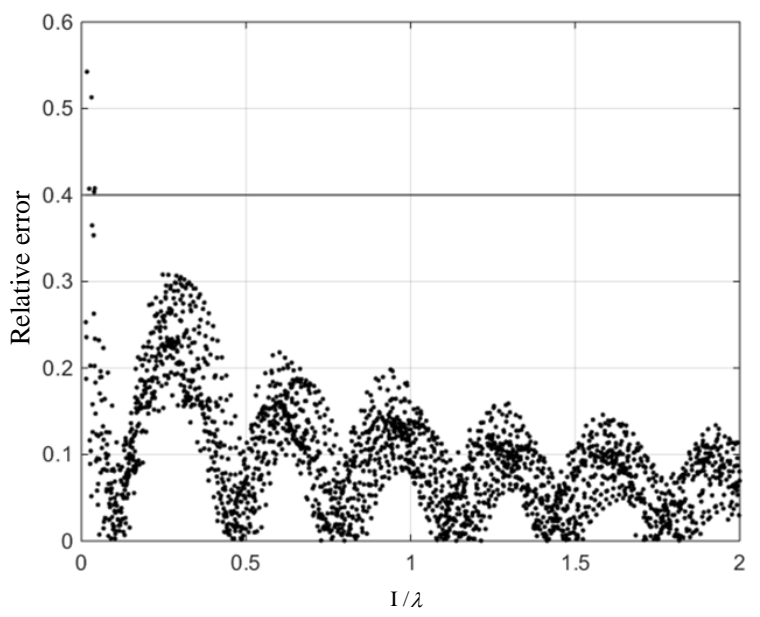

(c) $\varphi=30^{\circ}$ 


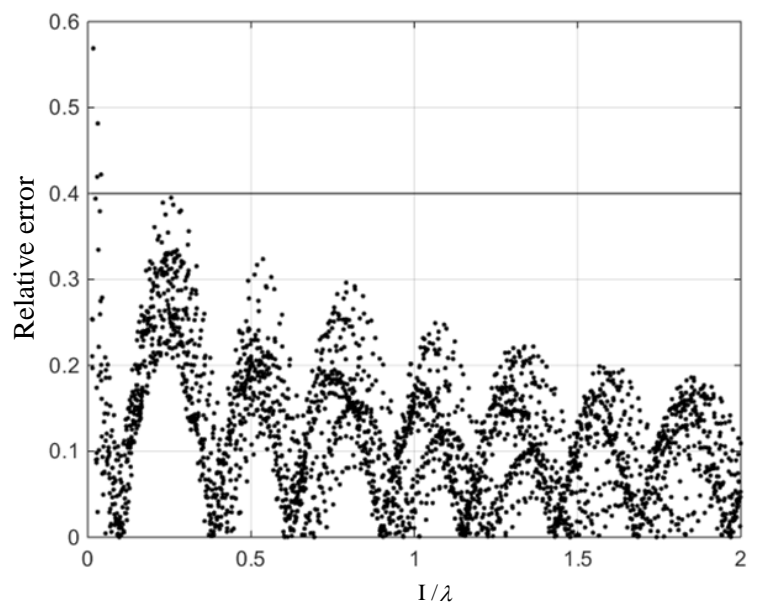

(d) $\varphi=60^{\circ}$

Figure 6. Variation of Relative Error Along With Relative Distance When the Oblique Incidence Occurs

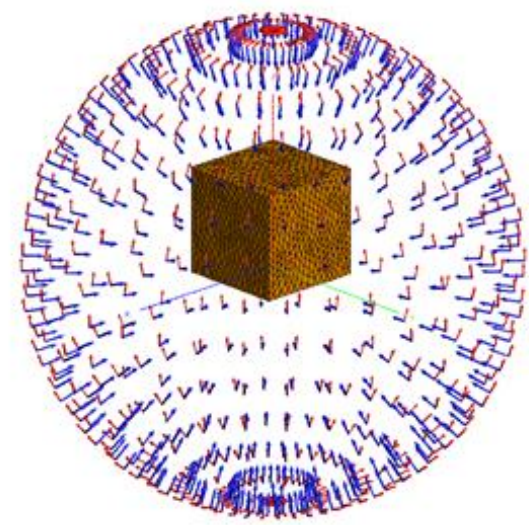

Figure 7. Simulated Reverberation Chamber Environment of Multi-Column Plane Wave Superposition

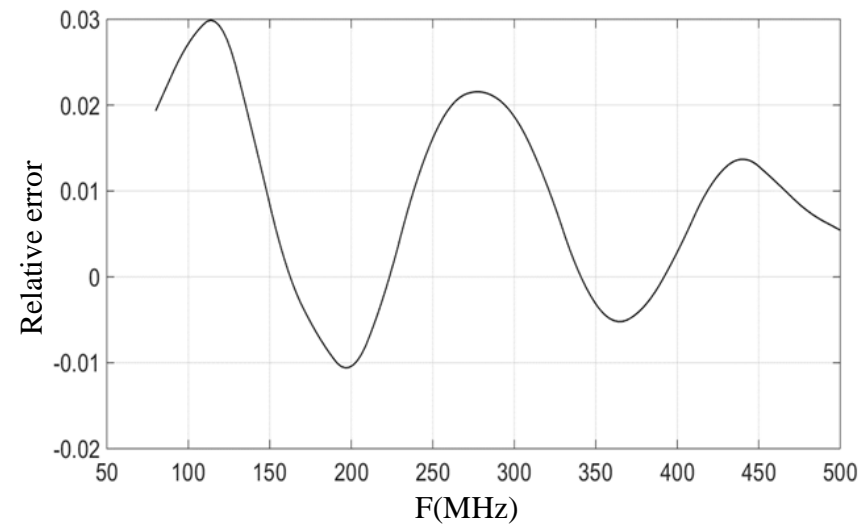

Figure 8. Variation of Relative Error of Test Point Along With Working Frequency 


\section{Selection of Measuring Position in Reverberation Chamber}

Hill.D.A theory deduces plane wave integral equation expression of field distribution in reverberation chamber after intensive mixing, statistical property of it can be described with plane angle spectrum of random variable [7], a certain number of random plane wave superposition is used by Franco Moglie to simulate electromagnetic wave spreading in different directions due to rotation of stirrer [3]. This article uses multi-column plane wave incident to simply simulate filed distribution in reverberation chamber and unit plane wave of direction angle and height angle which impose linear polarization as $10^{\circ}$ interval as shown in figure 7.

$l / \lambda=0.2$ point from relative distance of cube vertex angle is selected as test point, filed intensity module value $|E|$ and $\left|E^{\prime}\right|$ of this test point where is placed at back and forth of plane wave field, the relative error $\varepsilon$ of testing relative error is indicated as:

$$
\varepsilon=\frac{\left|E^{\prime}\right|-|E|}{|E|}
$$

Figure 8 shows testing relative error of selected point when working frequency is $80 \mathrm{MHz}$ to $500 \mathrm{MHz}$ using software simulation calculation, it can be seen that relative error appears to be an variation of damped oscillation with the increase of operating frequency, and relative error is as small as within 3\%, this is because reverberation chamber model established is ideal electromagnetic environment, there is an interaction and interplay between different incident direction and plane wave, relative error of it is smaller compared with the ones of single wave, simulation experiment has verified that test point selection may be applied to the measurement of environmental field intensity of test equipment in reverberation chamber.

\section{Conclusion}

This article is begun with vertical incidence of unit plane electromagnetic wave, good conductor cube is set as test equipment, simulating calculation concluded selection principle of test direction and test position when relative error is within $15 \%$, namely chiefly select the position of relative distance $l / \lambda \geq 2$ away from cube on $\theta=180^{\circ}$ test direction, when lower working frequency and experiment site restrictions are hardly to meet the conditions of above distance, test position of relative distance $l / \lambda=0.2$ away from cube on same test direction is selected, and verified test point selected of cube of different electrical size, which abides by this principle, may meet the relative error within $15 \%$. The condition of plane wave oblique incidence is inspected, when incident angle is selected as $\varphi=-30^{\circ}, \varphi=-60^{\circ}, \varphi=30^{\circ}$ and $\varphi=60^{\circ}$, relative error of field intensity value of test position, which abides by this principle, and actual environmental filed intensity value is calculated and can be guaranteed within $40 \%$, thus promoting it to reverberation chamber environment of random plane electromagnetic wave superposition, relative error of field intensity of the position and environmental field intensity is calculated when frequency is $80 \mathrm{MHz}$ and $500 \mathrm{MHz}$, it's required to guarantee that it meets the demand of experimental error and can be applied to actual experimental measurement.

\section{Acknowledgement}

The project of the Natural Science Foundation of Hefei University (Grant No. 12KY09ZR) 


\section{References}

[1] X. Li, Z. Lv and J. Hu, "XEarth: A 3D GIS Platform for managing massive city information", Computational Intelligence and Virtual Environments for Measurement Systems and Applications (CIVEMSA), 2015 IEEE International Conference on. IEEE, (2015), pp. 1-6.

[2] J. Yang, B. Chen and J. Zhou, "A Low-Power and Portable Biomedical Device for Respiratory Monitoring with a Stable Power Source Sensors, vol. 15, no. 8, (2015), pp. 19618-19632.

[3] G. Bao, L. Mi, Y. Geng and K. Pahlavan, "A computer vision based speed estimation technique for localizing the wireless capsule endoscope inside small intestine", 36th Annual International Conference of the IEEE Engineering in Medicine and Biology Society (EMBC), (2014).

[4] X. Song and Y. Geng, "Distributed community detection optimization algorithm for complex networks", Journal of Networks, vol. 9, no. 10, (2014), pp. 2758-2765.

[5] D. Jiang, X. Ying and Y. Han, "Collaborative multi-hop routing in cognitive wireless networks", Wireless Personal Communications, (2015), pp.1-23.

[6] J. Hu and Z. Gao, "Modules identification in gene positive networks of hepatocellular carcinoma using Pearson agglomerative method and Pearson cohesion coupling modularity", Journal of Applied Mathematics, (2012).

[7] D. Jiang, Z. Xu and Z. Chen, "Joint time-frequency sparse estimation of large-scale network traffic", Computer Networks, vol. 55, no. 15, (2014), pp. 3533-3547.

[8] J. Hu, Z. Gao and W. Pan, "Multiage Social Network Recommendation Algorithms and Similarity Network Evaluation", Journal of Applied Mathematics, (2013).

[9] M. Zhou, G. Bao, Y. Geng, B. Alkandari and X. Li, "Polyp detection and radius measurement in small intestine using video capsule endoscopy”, 2014 7th International Conference on Biomedical Engineering and Informatics (BMEI), (2014).

[10] G. Yan, Y. Lv, Q. Wang and Y. Geng, "Routing algorithm based on delay rate in wireless cognitive radio network", Journal of Networks, vol. 9, no. 4, (2014), pp. 948-955.

[11] Y. Lin, J. Yang and Z. Lv, "A Self-Assessment Stereo Capture Model Applicable to the Internet of Things", Sensors, vol. 15, no. 8, (2015), pp. 20925-20944.

[12] K. Wang, X. Zhou and T. Li, "Optimizing load balancing and data-locality with data-aware scheduling", Big Data, 2014 IEEE International Conference on. IEEE, (2014), pp. 119-128.

[13] L. Zhang, B. He and J. Sun, "Double Image Multi-Encryption Algorithm Based on Fractional Chaotic Time Series", Journal of Computational and Theoretical Nanoscience, vol. 12, (2015), pp. 1-7.

\section{Authors}

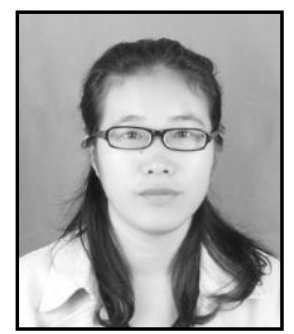

Zheng Juan, she is currently a lecturer in the Department of Electronics \& Electrical Engineering at Hefei University. Her research interest is mainly in the area of Computational electromagnetism, antenna design. She has published several research papers in scholarly journals in the above research areas and has participated in several projects. 